François Bonnet, "Les effets pervers du partage de la sécurité. Polices publiques et privées dans une gare et un centre commercial", Sociologie du travail, 50 (4), 2008, pp. 505-520

\title{
Les effets pervers du partage de la sécurité. Polices publiques et privées dans une gare et un centre commercial
}

\author{
François Bonnet ${ }^{1}$
}

\begin{abstract}
Résumé. Cet article s'intéresse aux acteurs du contrôle de la délinquance dans une gare et un centre commercial d'une grande ville française. L'implication croissante d'acteurs privés dans la mise en ouvre de politiques de sécurité dans les espaces publics suscite une littérature sur les dangers de la "privatisation". En fait, l'enquête de terrain montre que les acteurs non-étatiques (agents de sécurité, associations caritatives) remplissent des fonctions laissées vacantes par les policiers, comme la relation avec le public ou le recours à des politiques sociales. Dans ce partage de la sécurité, la police se concentre sur des missions purement bureaucratiques, ce qui affecte sa légitimité. L'approche historique permet de comprendre comment les mauvaises relations entre la police et les autres acteurs se sont développées.
\end{abstract}

Mots-clé : privatisation; sécurité; policing; sécurité privée; politiques sociales; médiation

\section{Introduction : la privatisation de la sécurité en question}

L'évolution du rôle et du périmètre de l'État au cours des vingt-cinq dernières années a nourri une littérature abondante en sciences sociales, paradoxalement plus centrée sur la réforme de l'État (Bézès 2005, 2006), sur la protection sociale (Palier 2005, Hassenteufel et Palier 2005) et sur les questions économiques (Jobert 1994, Le Galès 2003) que sur les fonctions dites régaliennes - l'armée, la police, la production du droit et de la monnaie - pourtant rudement éprouvées. Dans ce travail, j'aborde la question des transformations du travail d'État dans le domaine de la sécurité intérieure. Weber (1963) ayant défini l'État contemporain comme le groupement politique qui revendique avec succès le monopole de la violence physique légitime, la police a longtemps été perçue comme le seul acteur de la sécurité intérieure digne d'intérêt. L'implication croissante de forces de sécurité privées dans la production de l'ordre au concret

\footnotetext{
${ }^{1}$ frabonnet at gmail dot com, Observatoire Français des Conjonctures Economiques (Sciences Po), Center for Urban Research and Policy (Columbia University). Je remercie Hugo Bertillot, Ayda Hadizadeh, Patrick Le Galès, Naïma Makri et le comité de rédaction de Sociologie du travail pour leur lecture de versions précédentes de cet article.
} 
implique de s'interroger sur les modalités et les conséquences de cette transformation.

Cette question est souvent abordée sous l'angle de la privatisation. La «privatisation du contrôle» a été largement dénoncée par une littérature normative comme la fin de la sécurité comme bien collectif. Des auteurs prédisent l'avènement d'une société où les plus aisés pourront payer pour leur protection tandis que les plus pauvres subiront la répression des agents de l'État: « de plus en plus, les forces du marché (...) mettent un prix sur des aspects de la vie qui sont censés être des bienfaits universellement partagés dans une société civilisée le sentiment de sécurité, la liberté de mouvement, et la prévention du crime » (South 1994 : 230). Selon Paolucci (2003 : 168), la privatisation de la sécurité constitue le cœur même des politiques sécuritaires, car le sentiment d'insécurité serait généré par l'industrie de la sécurité privée. Sheerer et Hess (1997 : 124) prévoient que la sécurité est en passe de devenir le luxe des classes possédantes, qui se protègent des classes dangereuses dans les gated communities. Loader et Walker (2001) entendent montrer que le policing est un bien public, et ne saurait donc être confié à des mercenaires sans légitimité. En somme, cette littérature craint l'avènement d'une division du travail entre polices publiques et polices privées, les premières réprimant les pauvres et les secondes protégeant les riches. Bayley et Shearing (1996: 602) ont ainsi pu craindre que «[1]es sociétés démocratiques occidentales tendent inexorablement, nous le craignons, vers un monde à la Orange Mécanique, où le marché et le gouvernement protègent les riches des pauvres - l'un en barricadant et en excluant, l'autre en réprimant et en emprisonnant ».

Les théoriciens de la privatisation opèrent une idéalisation de la police publique, une idéalisation imprévisible au regard d'un demi-siècle de recherche sur la police. Aux Etats-Unis et au Royaume-Uni, les sociologues ont décrit l'identité policière comme le produit du recrutement de jeunes hommes des classes populaires avec un faible niveau d'instruction : un imaginaire viril, empli de préjugés sexistes, homophobes et racistes (Bowling et Foster 2002 : 1013). En France, Dominique Monjardet a montré que la police « est indissociablement : un instrument du pouvoir, qui lui donne des ordres ; un service public, susceptible d'être requis par chacun; une profession, qui développe ses intérêts propres » (1996 : 9). En tant qu'instrument de l'État, la police publique défend les intérêts de l'État, comme l'ordre public ou la distinction entre nationaux et étrangers. Si la police est inégalement répartie sur le territoire, ce n'est pas fonction des niveaux mesurés de la criminalité, mais de la présence ou non de sites jugés stratégiques par le pouvoir politique. La police n'est pas seulement un instrument de l'État, elle est aussi une grande organisation bureaucratique qui développe des logiques corporatives d'autonomisation et d'opacité (Monjardet 1996: 283-284). La logique d'autonomisation et de corporatisme qui joue au sein de la police conduit les policiers à considérer qu'ils ne doivent rendre des comptes qu'à leur hiérarchie. Dans la plupart des démocraties occidentales, les policiers considèrent que le travail noble est d'arrêter les délinquants. En conséquence, ils méprisent le community policing - l'idée selon laquelle des partenariats effectifs entre le police et la population peut jouer un rôle dans une réduction du crime et une légitimité renforcée de la police (Moore 1992: 123). En somme, il n'y pas de raisons de penser, à partir de la recherche en sciences sociales, que la police publique agisse 
de façon plus « démocratique » que les acteurs privés. Non seulement il est erroné de procéder à une idéalisation de la police publique, mais en plus, le terme de «privatisation» est inapproprié, car il empêche de voir l'essor des polices municipales ou des milices de citoyens, et est saturé de connotations normatives (Bayley et Shearing 2001, Roché 2004, Ocqueteau 2004).

Ce travail entend tirer les conséquences de l'implication des acteurs nonétatiques dans la production de l'ordre. Bien que ces acteurs, et particulièrement les acteurs privés, soient plus visibles aujourd'hui, l'État «passe [toujours] pour l'unique source du 'droit' à la violence » (Weber 1963 : 125). Le développement des polices municipales et des polices privées obéit toujours à une logique de subordination juridique et pratique à la puissance étatique. Analyser l'implication des acteurs non-étatiques dans la production de l'ordre ne signifie pas, selon le mot de Ocqueteau, d'essayer de mesurer les «mètres perdus de l'emprise de l'État». Il faut étudier «les définitions, les usages, les utilisations réelles des réformes impulsées, et les mettre en perspective avec les appropriations de sens et les pratiques réelles des acteurs publics et privés engagés dans la production de l'ordre local sur un territoire spécifique » (Ocqueteau $2004: 172$ ). C'est ce que l'on va faire en comparant la mise en œuvre de politiques de sécurité dans une gare et un centre commercial d'une grande ville de province.

A rebours des théories de la privatisation qui prédisent un policing plus inégalitaire et moins soucieux de ceux qui ne peuvent payer pour leur sécurité, ce travail montrer que le développement des acteurs privés a conduit à un partage de la sécurité : les policiers se focalisent sur les infractions légales et la formalisation des procédures judiciaires, et les agents de sécurité se concentrent sur le maintien de la civilité des relations sociales sur les espaces dont ils ont la charge. Or ce partage a eu des effets pervers sur la police. Il s'agit donc dans de comprendre la formation concrète des enjeux des acteurs impliqués et de reconstruire la rationalité de leurs pratiques. Après avoir présenté le cadre méthodologique de ce travail (2), ce travail étudie les questions de la relation des acteurs privé de la sécurité avec le public (3), de la dimension sociale de la production de l'ordre (4), et du repli sur elle-même de la police (5) - un repli à resituer dans une perspective d'histoire à moyen terme (6).

\section{Données : une enquête qualitative dans une gare et un centre commercial}

Le travail empirique qui fonde cette analyse repose sur deux monographies réalisées dans une gare et un centre commercial d'une grande ville française. L'enquête qualitative a essentiellement consisté à mener des observations directes et à réaliser des entretiens avec les acteurs de la sécurité sur chaque terrain. Recueillir des informations de première main est rendu nécessaire par l'absence de données quantitatives fiables et pertinentes pour le problème posé. Le choix de l'enquête ethnographique n'est cependant pas une solution de repli. Recueillir tous les points de vue permet de faire émerger une synthèse qui est différente de la somme des points de vue particuliers de chaque acteur. L'enquête doit donc aboutir à «une simplification du réel, en rupture partielle avec les interprétations et les visions ordinaires des acteurs du contexte d'action étudié » (Friedberg 1993 : 317). 


\begin{tabular}{|c|c|c|}
\hline Terrain & \begin{tabular}{|l|} 
Nombre \\
d'entretiens
\end{tabular} & Personnes interviewées \\
\hline $\begin{array}{l}\text { Gare } \\
\text { ferroviaire }\end{array}$ & $\begin{array}{l}26 \\
\text { entretiens, } \\
29 \\
\text { personnes } \\
\text { interviewées }\end{array}$ & $\begin{array}{l}\text { SNCF : le chef de gare (deux fois), deux employées } \\
\text { qui travaillent dans la gare, trois employés de la } \\
\text { Mission solidarité } \\
\text { Travailleur social : un travailleur social } \\
\text { Surveillance générale : le chef de brigade } \\
\text { (responsable régional), deux agents de maîtrise, un } \\
\text { syndicaliste, un agent, et un « focus group » avec } \\
\text { cinq agents } \\
\text { Commerçants : le président de l'association des } \\
\text { commerçants, deux gérants de magasin, et une } \\
\text { employée } \\
\text { Policiers : les deux chefs de poste et trois policiers } \\
\text { Syndicalistes : deux syndicalistes cheminots et un } \\
\text { syndicaliste du commerce (pour les magasins } \\
\text { présents dans la gare) }\end{array}$ \\
\hline $\begin{array}{l}\text { Centre } \\
\text { commercial }\end{array}$ & $\begin{array}{l}28 \\
\text { entretiens, } \\
31 \\
\text { personnes } \\
\text { interviewées }\end{array}$ & $\begin{array}{l}\text { Direction du centre : le responsable de la sécurité et } \\
\text { le médiateur } \\
\text { Commerçants : six directeurs, sous-directeurs ou } \\
\text { gérants ; trois responsables de la sécurité ou chefs } \\
\text { d'équipe de sécurité } \\
\text { Employés : sept employés } \\
\text { Policiers : le chef de poste et six policiers (dont deux } \\
\text { entretiens avec deux fonctionnaires) } \\
\text { Syndicalistes : quatre syndicalistes du commerce } \\
\text { (dont un entretien avec deux syndicalistes) et deux } \\
\text { de la sécurité. }\end{array}$ \\
\hline $\begin{array}{l}\text { Entretiens } \\
\text { transversaux }\end{array}$ & $\begin{array}{l}4 \text { entretiens, } \\
4 \text { personnes } \\
\text { interviewées }\end{array}$ & $\begin{array}{l}\text { J'ai par ailleurs interviewé le commissaire du } \\
\text { secteur, l'adjoint au maire en charge de la sécurité, } \\
\text { le responsable des Contrats Locaux de Sécurité, et } \\
\text { un journaliste local. }\end{array}$ \\
\hline
\end{tabular}

On a choisi d'étudier une gare et un centre commercial parce que ces espaces offrent un concentré de situations intéressantes pour notre problème théorique. Ces espaces sont circonscrits, ce qui facilite le recueil des données. Ils représentent un enjeu de sécurité pour une multitude d'acteurs susceptibles d'engager des ressources: la préfecture, la municipalité et les acteurs économiques. Ces acteurs financent différentes sortes de fournisseurs de sécurité : la police, des associations caritatives, et des agents de sécurité. L'intérêt de ces cas est de permettre d'analyser les relations entre ces différents acteurs. Il est cependant bien évident que ces cas sont singuliers. Il ne s'agit donc pas de faire comme si ce type de partenariat représentait un modèle ou une norme future mais de choisir un contexte particulier pertinent pour des questions spécifiques, en l'occurrence sur les relations entre acteurs publics et acteurs privés. Deux monographies ne peuvent prétendre couvrir à elles seules le champ de la recherche sur la police et la sécurité privée. Il faut donc considérer la gare et le centre commercial comme des concentrés de situations potentiellement conflictuelles où travaillent des organisations avec des enjeux et des logiques 
contradictoires, qui permettent de montrer des mécanismes concrets dont on a lieu de penser qu'ils ne sont pas spécifiques aux terrains investigués.

Bien qu'ils soient tous deux des espaces d'affluence, les gares et les centres commerciaux représentent des enjeux différents. Les gares sont des espaces stratégiques pour les États, puisque elles sont les points nodaux par lesquels transitent des millions de personnes. La question de la gestion des flux y est prépondérante. Dès 1845, «des services omniprésents de police et de contrôle » (Ribeill 1996: 58) sont institués par l'État, qui exige de contrôler l'affluence des voyageurs à une époque marquée par la psychologie des foules de Le Bon et la «hantise des foules non-ordonnancées » (Bowie 1996: 22). La police au XIXe siècle fait de la gare un «bastion défensif» (Ribeill : 52) : les chemins de fer sont entièrement clôturés, les cantonniers portent des sabres, il est interdit de ne pas attendre dans les salles d'attentes qui sont grillagées, et la mendicité ainsi que le démarchage sont strictement interdits. La police est toujours présente dans les grandes gares.

Les centres commerciaux sont différents. Leur attractivité dépend de leur capacité à fournir un environnement sûr aux clients (Rybczynski 1993, Cohen 1996, Goss 1993, Bickford 2000 et Jackson 1996). Ils sont plus souvent contrôlés par les seuls acteurs privés. Mais les très grands centres commerciaux sont presque des espaces publics, car ils ne sont pas fréquentés que par des consommateurs; ils sont aussi des lieux de vie. Pour contrôler les troubles à l'ordre public, les préfectures jugent irresponsable de ne pas être présente dans les grands centres commerciaux quand elles en ont les moyens. Comment s'organise, sur ces deux espaces, la production de l'ordre?

\section{Les agents de sécurité privée au contact de la clientèle}

Les gares et les centres commerciaux sont gérés par des entreprises commerciales dont les objectifs sont, in fine, la rentabilité de l'exploitation. En conséquence, la politique de sécurité mise en œuvre n'est qu'un élément d'une politique plus générale visant à accueillir le public dans des conditions qui maximisent la rentabilité commerciale de l'espace en question. Selon le mot de Sherman (1995: 111), «il n'est pas surprenant que lorsqu'un commissaire de Washington D.C. proposa aux commerçants asiatiques chroniquement dévalisés par les voleurs de fermer leurs magasins la nuit, la réaction fut un hurlement de protestation ». Les politiques de sécurité du centre commercial (3.1) et de la gare (3.2) sont mises en œuvres par les agents de sécurité privée.

\subsection{Les contraintes des agents de sécurité du centre commercial}

L'impératif premier d'un commerçant, ce n'est pas de se protéger du crime, mais de maximiser les ventes. C'est pourquoi l'impératif premier de la sécurité privée dans les centres commerciaux est de ne pas entraver la dynamique commerçante. Autant une situation pacifiée facilite la consommation, autant la présence excessive de dispositifs de sécurité est nocive, comme le souligne Flusty (2001 : 660) : "d'un côté, les usagers exigent la présence d'une protection visible et rassurante contre les rencontres imprévisibles et potentiellement déplaisantes avec l'altérité ; de l'autre, ils hésitent à vivre, sympathiser et dépenser leur argent dans l'anti-esthétique hostile de quelque chose ressemblant à un camp militaire ». 
Dans le centre commercial, les agents de sécurité des magasins ont deux missions : empêcher les voleurs de fuir et veiller à ce que le magasin ne soit pas le théâtre d'un désordre. Leurs missions sont compliquées par leur obligation professionnelle d'accueil courtois et discret de la clientèle. Parce qu'ils surveillent la clientèle, ils ne peuvent s'inscrire dans une rationalité exclusive de « chasse à la démarque » ou d'ordre public; ils doivent composer avec la logique commerciale du magasin qui les emploie.

La sécurité, elle doit être efficace, courtoise et polie, et le client il pense "c'est la Fnac, ils sont sympas». On est là pour servir notre client. (...) Le but c'est que les gens reviennent acheter. (Responsable de la sécurité, grande surface loisir, centre commercial)

$\mathrm{Si}$, suite à une suspicion, une personne est interpellée à tort, le magasin est tenu pour responsable de l'humiliation qui a été faite au client. Les agents de sécurité préfèrent donc laisser partir une personne suspecte que de créer un conflit entre le client et le magasin. Les agents de sécurité - ceux des magasins comme ceux de la direction du centre - ont pour mission de ne faire qu'un usage exceptionnel de la force et de recourir au maximum à la patience et au dialogue (voir aussi Hug 2000 : 104). Cette injonction est motivée par le souci d'éviter le spectacle de la violence à la clientèle et de ne pas attiser les conflits avec les adolescents en leur donnant une raison ou un prétexte pour mener des représailles contre les magasins. Le spectacle de la violence et la dégradation de la réputation qui s'ensuit sont nuisibles à l'activité commerciale. Les circonstances d'une rixe peuvent amener la clientèle à se placer du côté de la «victime » des agents de sécurité. Dans tous les cas, une atmosphère sereine et propice à la consommation exclut l'idée de violence.

(C'est quoi des agents très bien ?) C'est un agent très diplomate avec un très bon relationnel, pas une brute. (...) Avant les agents étaient en jean et en pull, maintenant ils sont en pantalon veste et cravate, et ça a contribué au changement d'image du centre commercial auprès des clients. Ils ont bien joué le coup. Nos agents ont eux une tenue correcte depuis longtemps. Le but c'est pas de faire de la répression mais de développer un respect par rapport au relationnel et au diplomatique. (Directeur, fast-food, centre commercial)

Les agents de sécurité dépendent de sociétés dont les services sont achetés par les grandes surfaces du centre commercial. En conséquence, les agents de sécurité mettent en œuvre une «sécurité » définie selon les enjeux commerciaux de ceux qui achètent le service. Le souci de la clientèle est fondamental dans les instructions que les commerçants donnent aux agents de sécurité privée. C'est le payeur, c'est-à-dire le magasin, qui définit les règles, le cadre et les contraintes de l'exercice de la sécurité. La raison d'être d'un magasin est de faire des bénéfices en vendant le maximum de produits; la protection contre le vol n'est qu'un moyen pour limiter les pertes qui ne doit pas empêcher les clients de consommer. Les commerçants demandent donc expressément aux agents de sécurité de tenir compte des préoccupations commerciales, même si celles-ci rentrent en conflit avec les préoccupations sécuritaires. Les enjeux 
commerciaux du commanditaire de sécurité se retrouvent de la même façon dans les gares.

\subsection{La prise en compte des voyageurs à la SNCF}

A la SNCF, le service chargé de protéger l'entreprise contre les actes de malveillance s'appelle la Surveillance générale, surnommée la « Suge ». La Suge est le seul département de la SNCF qui ait vu ses effectifs croître dans les dix dernières années, alors que le nombre des salariés a fondu de 515000 en 1945 à 180000 aujourd'hui (Guélaud 2002: 127). Le cas de la Suge illustre la dépendance des acteurs privés de la sécurité envers les enjeux de leurs employeurs. Dans la perspective de l'ouverture à la concurrence, la SNCF a radicalement changé les missions de la Suge pour en faire un service supplémentaire pour les voyageurs.

Avant les années 1990, l'activité de la Suge était surtout orientée vers les problèmes en interne - l'argent qui disparaît dans les caisses, le vol de matériel ou le sabotage. Le seul rapport que les agents de la Suge avaient avec les autres employés de la SNCF était un rapport de surveillance et de répression. La Suge a entretenu avec les syndicats dominants de la SNCF (au premier rang desquels la CGT) des relations conflictuelles. Le surnom commun de la Suge était «la cinquième », abréviation de «la cinquième colonne ${ }^{2}$. L'évolution de la Suge a commencé dans la région parisienne au début des années 1990. Le moteur de cette évolution a été le mécontentement croissant du personnel SNCF et des voyageurs vis-à-vis des dégradations, des vols et des violences croissants dans les RER et les trains de banlieue. Pour répondre à la peur de la délinquance, la direction centrale de la SNCF a réorienté le travail de la Suge en lui assignant comme tâche prioritaire la sécurisation des trains et des gares. Cette réorientation a transformé le travail des agents. La plus spectaculaire de ces transformations a été le passage du travail en civil au travail en uniforme. Le passage à l'uniforme interdit tout un ensemble d'activités autrefois quotidiennes : les planques, les filatures, c'est-àdire la partie « répressive » du travail.

L'ancienne Suge, en civil, n'avait aucune visibilité pour la clientèle. Quelle que fut son efficacité dans la chasse aux contrevenants, elle n'en avait aucune aux yeux des voyageurs, puisqu'elle travaillait dans la discrétion. Son utilité pour la SNCF est donc devenue presque nulle quand les enjeux ont changé et que les objectifs ont été redéfinis. D’une logique de répression des délinquants la Suge est passée à une logique de présence et de dissuasion : il faut patrouiller en uniforme («montrer du bleu », selon l'expression locale) dans la gare, que les agents se voient, que leur travail se fasse au vu et au su de tous. Les autres cheminots cessent d'être des suspects potentiels et deviennent des collègues qu'il faut protéger comme la clientèle. La plupart des agents qui ont connu l'ancienne Suge n'apprécient pas leur nouveau statut. Ceux qui se sont engagés pour faire des planques et agir en civil vivent le passage en tenue comme une régression. Faire des rondes dans la gare expose les agents à remplir des fonctions qui n'étaient pas traditionnellement les leurs, comme le renseignement des voyageurs, qui est une

\footnotetext{
${ }^{2}$ Pendant la guerre d'Espagne, on demanda au général franquiste en charge de la prise de Madrid laquelle de ses quatre colonnes allait pénétrer dans la ville la première, il répondit que sa cinquième colonne était déjà dans les murs.
} 
mission dévolue aux agents d'accueil. Le passage à la tenue et aux rondes implique d'indiquer aux voyageurs où sont les guichets, les quais ou les toilettes.

Ça a bien arrangé l'entreprise, elle a trouvé ses billes. Elle mettait de l'argent, on était en civil et les gens nous voyaient pas. Mais en tenue on nous voit. Les gens voient que la SNCF investit pour la sécurité. (Agent de maîtrise 1, Surveillance générale SNCF)

Les transformations des missions de la Suge s'inscrivent dans une logique commerciale et traduisent les enjeux commerciaux en matière de sécurité de la SNCF. Pour la direction des gares à la SNCF, la clientèle doit être rassurée quand elle montre des appréhensions. Comment montrer que l'entreprise agit pour la sécurité des voyageurs? La nouvelle Suge, en faisant des rondes en uniforme, s'expose au regard de tout un chacun, et c'est cela qui est recherché par la SNCF : le sens premier de ces rondes n'est pas de dissuader d'éventuels délinquants, mais de rassurer les voyageurs et de les guider dans la gare.

Dans la gare comme dans le centre commercial, l'activité des acteurs privés de la sécurité est structurée par les enjeux de leurs commanditaires. En l'occurrence, les entreprises commerciales ont besoin d'une sécurité qui soit au service du client et qui ne nuise pas à la profitabilité. Le souci de la clientèle rentre directement en contradiction avec les théories selon lesquelles la sécurité privée ne protège que les riches aux dépens des pauvres. Comme on le verra plus loin, la sécurité qui est mise en œuvre n'est donc pas la même que celle que promeut la police. Le cas est plus flagrant quand on prend en compte les efforts fournis par les acteurs non-étatiques pour gérer la dimension sociale de la sécurité.

\section{Gérer la dimension sociale de la sécurité}

Par « dimension sociale », on fait référence aux problèmes de sécurité qui ne sont pas purement délictuels au sens juridique, comme la conflictualité des rapports entre les forces de l'ordre et les adolescents issus de l'immigration au centre commercial ou la présence de sans-abri dans la gare. Dans les deux cas, les acteurs non-étatiques investigués recourent à des stratégies non répressives qui ne font pas partie du répertoire de l'action policière : politiques de médiation au centre commercial (4.1) et politiques sociales à la gare (4.2).

\subsection{La politique de médiation au centre commercial}

Pour les commerçants, les vols et les désordres sont deux problématiques très différentes. Les vols constituent une préoccupation de premier ordre, mais ils sont invisibles et ne dissuadent pas les clients de venir. En revanche, la tranquillité court perpétuellement le risque d'être troublée par des désordres, comme des adolescents turbulents ou des bagarres. Les désordres sont mauvais pour le commerce car ils nourrissent une réputation d'insécurité. Le centre commercial a connu, en 1998, une émeute de grande ampleur impliquant principalement des adolescents issus de l'immigration maghrébine et venant des banlieues populaires. Cette émeute a provoqué une transformation radicale de la politique de sécurité du centre commercial. Le souvenir de l'émeute de 1998 a incité la direction du centre et les commerçants à tenir compte du risque que de nouveaux incidents ne dégénèrent en affrontements avec la police et en vandalisme à grande échelle. 
Pour apaiser les conflits avec les forces de l'ordre (publiques et privées), la direction du centre a recruté un médiateur. Il s'agit d'un professeur d'arabe, personnalité d'une radio communautaire, qui vient au centre l'après-midi, quand les adolescents issus de l'immigration maghrébine arrivent. Bien qu'il s'en défende, il est clair que le médiateur a pour mission prioritaire de «calmer le jeu » avec ces adolescents. Il se déplace dans le centre avec une radio et reçoit des appels quand un groupe d'adolescents devient trop turbulent. Il se rend alors sur les lieux pour traiter les différends sur un mode non-conflictuel. Soit le cas d'une dispute entre un adolescent et un commerçant. Le médiateur et les agents de sécurité du centre interviennent. Le médiateur isole alors l'adolescent dans les coursives du centre (les couloirs extérieurs fermés au public), afin que son dialogue ne soit pas perturbé par un tiers. Son souci est de calmer l'individu sans provoquer l'ire du commerçant (qui pourrait s'insurger contre le «laxisme» du centre). La distinction recherchée entre le médiateur et les agents de sécurité est significative de la volonté du centre de prévenir les problèmes par la négociation plutôt que par la répression des désordres.

Outre l'embauche du médiateur à la suite de l'émeute de 1998, la direction du centre a recruté, des agents de sécurité eux-mêmes issus de l'immigration et des quartiers pauvres afin de favoriser le dialogue entre adolescents et forces de l'ordre et de minimiser le risque des accusations de racisme de la part des agents de sécurité. Une politique trop répressive vis-à-vis des délinquants pourraient les amener à se venger en multipliant les vols. Ils pourraient même commettre des dégradations, ou des actes de malveillance de nature à compromettre la sécurité de la clientèle. La peur des représailles est associée aux adolescents issus de l'immigration maghrébine. Le souvenir de l'émeute est encore vif chez les commerçants et aucun n'a envie de voir sa devanture saccagée et son magasin pillé. La peur des représailles est accentuée par la diffusion de stéréotypes sur la solidarité familiale dans les fratries maghrébines ou sur la conception méditerranéenne de l'honneur.

(Mais vous portez plainte à chaque fois ?) Non, parce qu'ensuite les racailles elles ont la mort contre nous, alors... Et puis de toutes façons au bout de deux heures ils sont relâchés, alors à quoi ça sert? Du moment qu'on récupère la came [les biens volés]. (Vendeur, petite boutique textile, centre commercial)

Le travail de l'équipe de sécurité du centre est pris dans une double exigence. Il s'agit de calmer les adolescents issus de l'immigration maghrébine pour que le centre soit convivial, selon les standards de la classe moyenne, tout en évitant le risque d'émeutes et de vandalisme de masse. Mais les adolescents sont aussi des clients. C'est pour cette raison que les commerçants et la direction du centre ont adopté une politique de sécurité compréhensive - et pas seulement par peur d'une nouvelle émeute. Certes, d'aucuns causent des troubles, commettent des petits délits, ou flânent bruyamment en groupe dans les allées du centre. Mais ni la direction ni la plupart des commerçants ne voudraient que ces clients disparaissent. Les nuisances sont compensées par le pouvoir d'achat des adolescents, ce qui justifie une politique de sécurité accommodante qui veille à ménager les susceptibilités. Cette politique accommodante, fondée sur la 
médiation, semble efficace : depuis 1998, il n'y a pas eu d'émeute. La dimension sociale des politiques de sécurité est plus évidente encore dans les gares.

\subsection{Le traitement social du problème des sans-abri dans la gare}

En France, la crise économique du début des années 1990 a provoqué une forte augmentation du nombre de sans-abri. Les gares attirent les marginaux. Historiquement, leur prise en charge a hésité entre «la potence ou la pitié » (Geremek 1987, Damon 2003), entre la répression et l'assistance. Dans les années 1990 , le problème des «personnes en errance » (selon la terminologie officielle de la $\mathrm{SNCF}$ ) a suscité une réponse en termes de politique sociale.

La présence des sans-abri dans la gare est considérée par les voyageurs, par les commerçants et par les cheminots comme une nuisance. Le spectacle de la misère, les déjections et les débordements alcoolisés inquiètent et dérangent les usagers. La SNCF, qui est responsable sur son territoire, pourrait choisir une solution coercitive : utiliser sa Surveillance générale pour harceler les sans-abri, les faire circuler autant que possible, leur signifier clairement qu'ils ne sont pas les bienvenus dans la gare. C'est en général de cette façon que procèdent les centres commerciaux - avec des entrées beaucoup plus faciles à surveiller.

En 1998, dans la gare étudiée, la SNCF envisage de «déloger toutes les personnes indésirables ${ }^{3}$ «qui sont stationnées à proximité des commerces de la gare. Une telle action aurait consisté en l'intervention des agents de la Suge. Mais elle se heurte aux contraintes juridiques : les agents de la Suge ont le droit de faire circuler les sans-abri, mais ils n'ont pas le droit de les empêcher de revenir. En conséquence, les agents de la Suge peuvent à la rigueur harceler les sans-abri, mais ce type de tactique montre ses limites avec des individus qui « habitent» autour de la gare depuis des années. Que peut valoir une injonction de circulation face à des années de routines de survie sur un espace donné ? Un comité de suivi, piloté par la Mission Solidarité de la SNCF et composé de représentants de la SNCF, de la Ville, de la DDASS, de la police et des commerçants de la gare, décide de commencer l'opération de «nettoyage » de la gare en agissant en deux temps. D'abord, des travailleurs sociaux doivent mener une expertise et d'établir un contact avec les sans-abri. Ensuite, les travailleurs sociaux doivent proposer des solutions de relogement aux sans-abri, afin de leur faire quitter la gare de façon définitive. Cela soulevait de nombreux problèmes compte tenu de l'ambivalence de l'attitude des usagers de la gare.

Mettre en œuvre le projet de la Missions solidarité suppose, pour la $\mathrm{SNCF}$, de ne pas créer un précédent qui fasse penser aux sans-abri de la région qu'un séjour à la gare est la meilleure façon de trouver une aide institutionnelle durable. En d'autres termes, il s'agit d'aider les sans-abri, pour les faire partir, sans en attirer d'autres : «qu'ils (s'en) sortent» (Soutrenon 2001). Reloger les sans-abri consiste à les convaincre d'abandonner la gare et d'accepter de vivre dans un appartement ou dans un foyer. Cela est plus compliqué qu'il n'y paraît, parce que les sans-abri ont développé des routines et des sociabilités dans les lieux qu'ils fréquentent ; 13 sans-abri ont « une attache forte au site ${ }^{4}$ «. Les nuisances qu'ils occasionnent dépendent de leur taux d'alcoolisation et des vicissitudes de

\footnotetext{
${ }^{3}$ Document interne SNCF, 16 avril 1998

${ }^{4}$ Document interne de la Municipalité, mai 1999
} 
leur vie relationnelle («fusion ou embrouille entre les membres du groupe ${ }^{5} \ll$ ). La présence des travailleurs sociaux et leurs liens avec les sans-abri entraînent une diminution des nuisances avant même que la phase de relogement ne commence. Les commerçants, deux mois après le début de l'expertise, expriment leur satisfaction. La perspective de la « solution de secours »- l'expulsion autoritaire des sans-abri - s'éloigne.

Six mois plus tard, la plupart des sans-abri « historiques » ont été relogés par les associations. Pour parvenir à ce résultat, les travailleurs sociaux doivent s'occuper individuellement de chaque sans-abri, le convaincre de partir, lui faire accepter le relogement, tenir compte de ses pathologies physiques et mentales. Allant au-delà de leur mission initiale, les travailleurs sociaux cherchent maintenant à faire le lien entre les sans-abri et le monde institutionnel en facilitant les démarches administratives ${ }^{6}$. Pour empêcher que de nouveaux sans-abri ne viennent s'installer, la Suge et la police mènent une politique systématique de découragement des nouveaux arrivants. Le succès de l'opération convainc même l'association des commerçants de la gare de participer au financement des travailleurs sociaux. La stratégie du traitement social de la misère dans la gare ne doit pas seulement aux considérations humanitaires des travailleurs sociaux et des employés de la Mission solidarité de la SNCF, mais aussi au calcul rationnel de la direction de la gare à la SNCF et des commerçants. Dans cette optique, les travailleurs sociaux financés par la Municipalité et la SNCF font partie intégrante des acteurs impliqués dans la production de l'ordre.

Les deux cas de la gare et du centre commercial montrent comment les acteurs non-étatiques peuvent développer des solutions originales et efficaces quand ils sont confrontés à des problèmes de société. Les agents de sécurité issus de l'immigration et le médiateur dans le centre commercial, les travailleurs sociaux dans la gare n'ont pas été recrutés pour servir de simple caution. Leur travail est de remédier à des situations complexes où le recours à la coercition pourrait avoir des conséquences néfastes. Les théories de la privatisation de la sécurité, qui spéculent sur le développement de logiques inégalitaires et répressives liées à l'implication des acteurs non-étatiques, ne permettent pas de rendre compte des réalités empiriques: les partenariats public-privé peuvent donner lieu à l'émergence de politiques sociales. Le souci du public et l'inventivité des stratégies qui caractérisent ces acteurs non-étatiques contrastent avec le repli sur soi de l'institution policière.

\section{Le repli de la police}

L'examen du travail de la police montre, de façon cohérente avec la littérature sur le sujet, que les policiers se focalisent sur des priorités spécifiques comme les contrôles d'identités (5.1) et se refusent à coopérer avec les agents de sécurité qui travaillent sur le même espace (5.2).

\footnotetext{
${ }^{5}$ Collectif des Associations : X, Y, Z, «Premier bilan d'étape de l'action éducative engagée sur le site gare $* * *$ », juin 1999

${ }^{6}$ Collectif des Associations : X, Y, Z, « Troisième bilan d'étape de l'action éducative engagée sur le site gare $* * * »$, novembre 1999
} 


\subsection{Les priorités spécifiques du travail policier}

Dans le centre commercial, une grande partie du travail des policiers consiste à « judiciariser ${ }^{7} »$ les interpellations menées par les services de sécurité privée des magasins. Cela signifie que la grande majorité des interpellations ne sont pas à l'initiative des policiers, et que leur travail est de régulariser et de formaliser l'activité des agents de sécurité privée selon les canons de la procédure pénale. Schématiquement, les agents de sécurité surveillent et appréhendent, les policiers prennent les dépositions et les plaintes, et font les placements en garde à vue.

Dans la gare, les policiers ont deux missions principales : les patrouilles Vigipirate (avec des militaires) et les contrôles des personnes suspectes. Le problème pour eux n'est pas de parvenir à un objectif qualitatif de «tranquillité » dans la gare mais de fournir des résultats à la hauteur des objectifs chiffrés (nombre d'heures de patrouilles et personnes arrêtées), particulièrement depuis 2002. Les objectifs concernent les déviances qui constituent des enjeux particuliers pour l'État, comme le séjour irrégulier sur le territoire ou le trafic de stupéfiants.

On fait les chiffres ce matin. (Les statistiques?) Oui. (Et alors, c'est comment?) ça va bien, mais ici c'est un bon poste pour travailler. Ici, avec le stationnement... c'est complètement anarchique, les gens se garent n'importe comment pour poser des gens à la gare. Ici on est bien... Et puis avec [tel article de loi], (Schengen?) Oui, Schengen, on contrôle qui on veut! Alors on trouve des trucs. Drogue, situation irrégulière, fiches de recherche... Vous savez, on disait délit de sale gueule avant, mais ici c'est un peu ça, vous avez quelqu'un qui vous revient pas, un regard fuyant... On prend qui on veut! Si il passe devant le poste, baisse la tête, n'importe quoi, on le prend, on le contrôle, on le passe aux fiches de recherche. (Policier 4, gare)

Les missions de dissuasion et la pratique des contrôles permettent aux policiers d'être «efficaces » en fonction des critères prédéfinis de l'efficacité. Les policiers français tendent à privilégier la répression de la délinquance aux dépens du contact avec la population (Monjardet 1996, Roché 2005). Par exemple, dans la gare, les agents de la Suge et les cheminots disent rencontrer toutes sortes de problèmes avec les voyageurs. Outre les fraudeurs, il y a les gens perdus et stressés qui agressent les agents de la $\mathrm{SNCF}$, ceux qui ont peur de rater leur train, ceux qui ne veulent pas se soumettre à une mesure de filtrage à l'embarquement, et les victimes de ruptures de correspondance qui décompensent sur la personne de l'accueil. Pour les policiers, les nuisances dans la gare diffèrent. Les «bandes de jeunes », les pickpockets, les trafiquants de drogue, les étrangers en situation irrégulière et les personnes recherchées importent plus que les voyageurs agressifs. Les seuls voyageurs qu'ils voient sont ceux qui viennent porter plainte pour vols de bagage. La conception de la sécurité comme lutte contre la délinquance éloigne les policiers d'une prise en compte de ce que cheminots, commerçants et voyageurs considèrent comme les nuisances réelles.

\footnotetext{
${ }^{7}$ Christian Mouhanna, «Une police de proximité judiciarisée », Déviance et Société, vol. 26, n²,
} 2002, pp. 163-182 
5.2 Une absence de coopération avec les acteurs privés et un sentiment de défiance

Une des aspects du repli de la police est son manque de confiance et son refus de coopérer avec les autres acteurs de la sécurité. Dans la gare, les agents de la Suge évoquent une concurrence entre services sur les «affaires » (les flagrants délits, les enquêtes, la partie noble du travail) et une «jalousie » attribuée aux policiers, qui envieraient l'absence de prérogatives judiciaires des agents de la Suge. En retour, les policiers moquent la dimension « relation avec la clientèle » du travail de la Suge et font comprendre que cette concurrence ne se traduit pas par de la coopération.

Ben à mon niveau y'en a aucune, de coordination avec la police, voilà, ce que j'ai toujours connu c'est une certaine jalousie. (...) Les gardiens du poste, à chaque fois, ça leur prend une heure et demie, quoi. Faire la saisine, faire appel à un OPJ, prendre la plainte (...). Donc ça fait énormément de paperasse, alors que nous on a fait l'affaire, on leur a ramené et on est parti. (Agent, Surveillance générale SNCF)

Mais on n'a pas d'échanges d'infos ou quoi que ce soit, on n'a pas d'infos à leur donner, et ils n'ont pas d'infos à nous donner. (Policier 5, gare)

Dans le centre commercial, les relations entre police publique et privée sont bien plus conflictuelles. Pour la direction du centre commercial, une part importante du travail des agents de sécurité issus de l'immigration consiste à empêcher jeunes et policiers d'interagir, afin de limiter le potentiel de situations conflictuelles. En retour, les policiers mettent en cause l'honnêteté des agents de sécurité.

(Les vigiles du centre, ça se passe comment avec eux ?) Assez mal. Ça se passe, quoi. Ils sont pas recrutés comme il faudrait. Ils sont issus des quartiers où sont issus les délinquants. Donc ils négocient. (...) (Vous coopérez avec les agents du centre ?) Non, on n'échange aucune information, elle serait mal employée, il faut surtout pas qu'ils sachent comment on fonctionne. (Vous avez des doutes sur leur honnêteté ?) Oui. Enfin honnêteté je sais pas mais sur leur professionnalisme, oui. Ils ont laissé partir des délinquants, ils nous ont envoyé à gauche alors que le gusse était parti à droite, ensuite ils ont eu bien du mal à le justifier. (Policier 6, centre commercial)

Le repli sur soi de la police dans la production de l'ordre se comprend par l'hostilité que ressentent les policiers à leur encontre et qui les conduit à privilégier une forte cohésion interne et une faible ouverture envers les autres acteurs de la gare et du centre commercial. Les policiers se plaignent que la population ne leur témoigne pas la reconnaissance qu'ils estiment méritée, et que la justice ne soit pas aussi sévère qu'ils le souhaiteraient. Les policiers de la gare et du centre commercial ont en commun de projeter une image de la police dans laquelle il y a un intérieur protégé et réconfortant (le poste de police, les collègues, l'institution) et un extérieur hostile qui au mieux ne reconnaît pas la valeur de leur travail et au pire les exècre. 
Te faire traiter d'enculé et de fils de pute tout la journée, tant que tu portes pas la tenue, tu sais pas ce que c'est. (Policier 2, centre commercial)

Aujourd'hui il fait pas bon être flic. (...) Si j'avais une vie dans le futur, je le referais plus. Si on a des enfants, faut dire aux enfants de pas dire que les parents sont flics, sinon ils se font tabasser. (Policier 3, centre commercial)

Les policiers privilégient la dimension répressive de leur activité, au point de se désintéresser de ce qui peut se passer sur l'espace dont ils ont la charge et de mettre en œuvre des stratégies stéréotypées à finalité bureaucratique. Ils illustrent la dérive mise au jour par Wilson et Kelling (1982) : la police tend à se focaliser sur le respect des procédures, sur la mise en œuvre de normes juridiques (law enforcement), oubliant ipso facto de s'intéresser aux problèmes tels que les citoyens les ressentent (order maintenance). A l'échelle du centre commercial et de la gare, cela signifie que la police se focalise sur la mise en forme bureaucratique du travail de la sécurité privée et sur des missions sans rapport réel avec les besoins des usagers ou des entreprises commerciales ; reste à comprendre comment cette situation est advenue.

\section{Comprendre le repli de la police : deux niveaux d'explication}

Dans les sections précédentes, on a vu comment polices publiques et privées se partageaient la sécurité ; on va maintenant expliquer comment ce partage s'est construit dans le temps. A aucun moment l'État n'a « décidé » de réduire ses missions, et il n'a pas non plus incité au développement des sociétés de sécurité privée. Dans la gare comme dans le centre commercial, les acteurs privés ont dû réagir face à l'évolution de la délinquance.

Dans la gare, les voyageurs ont commencé à se plaindre de la petite délinquance à partir des années 1990. La réputation du transport ferroviaire a été affectée, notamment dans les trains de banlieue. La SNCF s'est tournée vers les autorités policières pour demander un renforcement des moyens humains, mais la police est une institution qui ne peut, à court terme, ni augmenter ses coûts, ni changer l'allocation de ses moyens ${ }^{8}$. Comme décrit précédemment, la SNCF a donc modifié les missions de son service de sécurité interne (la Suge) pour que la priorité soit donnée au rétablissement de la confiance des usagers. L'implication nouvelle de la Suge dans le traitement de la petite délinquance à contraint les policiers présents à augmenter la part procédurale de leur travail, et donc à diminuer la part du travail de terrain. La concurrence entre policiers et agents de la Suge sur les mêmes affaires s'est traduite par une détérioration des relations entre ces acteurs. Le changement s'est donc accompli de manière incrémentale : chaque perturbation de l'équilibre existant a entraîné des réponses pertinentes à court terme des acteurs concernés, ces réponses ont entraîné à leur tour de nouvelles perturbations qui ont commandé de nouvelles réponses, selon une

\footnotetext{
${ }^{8}$ Le statut de fonctionnaire de police et les modalités de recrutement de la police nationale interdisent une évolution importante du nombre de policiers à court terme. L'évolution des effectifs au niveau local ne dépend donc, à court terme, que de la réaffectation de moyens humains. Ces réaffectations sont contrariées par le statut des agents, qui les protègent des mutations intempestives, et par les pressions politiques locales : l'opération consistant diminuer les effectifs à un endroit pour les augmenter ailleurs ne manque pas de susciter des résistances. Voire Monjardet (1996).
} 
logique classique dans la sociologie des politiques publiques (Lindblom 1959, Simon 1982). Parce que les acteurs ne disposent que d'une information limitée et de peu de temps pour la réflexion, ils ne peuvent mettre en œuvre des stratégies élaborées à long terme. Ils sont constamment confrontés à de nouvelles contraintes et se doivent trouver les solutions les moins pires dans le laps de temps le plus réduit, des solutions dont ils ne peuvent prévoir les conséquences.

Au centre commercial, l'évolution est plus directement liée à la question structurelle des relations entre police et jeunes issus de l'immigration. Au début des années 1980, le centre commercial n'employait des agents de sécurité que pour répondre aux dispositions réglementaires sur les «espaces recevant du public » et sur les dispositifs anti-incendies. Les magasins employaient des agents de sécurité pour se prémunir des vols. Quand, au cours des années 1990, les incidents impliquant des adolescents issus de l'immigration se sont multipliés, les commerçants et la direction du centre commercial se sont tournés vers la police, qui, comme dans la gare, ne pouvait augmenter ses effectifs pour des raisons institutionnelles. L'émeute de 1998, qui a été réprimée par un important contingent de CRS, n'a fait que contribuer à la mauvaise réputation du centre commercial. Face à la dégradation du chiffre d'affaire des magasins fuis par leur clientèle traditionnelle, la direction du centre commercial a décidé d'embaucher un médiateur et des agents de sécurité eux-mêmes issus de l'immigration. Comme on l'a vu, les policiers ne font pas confiance aux agents de sécurité. Le cas du centre commercial implique de prendre en compte le contexte structurel des tensions entre jeunes issus de l'immigration et policiers qui ont cours sur tout le territoire français (voir entre autres Dubet 1987, Lepoutre 1997, Bachmann et Le Guennec 1997, Jobard 2002, Macé et Peralva 2002).

En somme, deux niveaux d'explication permettent de comprendre le repli sur soi de la police. Le premier niveau d'explication repose sur une logique micro de l'enchaînement de mécanismes, où les conséquences non-intentionnelles de décisions prises sous contrainte permettent de comprendre comment localement, les policiers en viennent à se priver de toute opportunité de répondre aux attentes de la population. Les policiers se sont dans un premier temps bien accommodés de l'arrivée de nouveaux agents sur lesquels ils continuaient à exercer une tutelle juridique. L'augmentation du nombre des agents de sécurité permettait à l'État de ne pas avoir à engager plus de policiers. Mais la dynamique de la situation a eu des conséquences non-intentionnelles: les policiers se sont coupés de la population, ce qui a nuit à la légitimité de l'institution policière.

Mais l'histoire serait incomplète si on ne replaçait pas la logique micro dans une logique macro des institutions et dans un contexte social donné, où l'histoire des émeutes urbaines et de leur répression au niveau national fournit un cadre de références qui contraint fortement les comportements de policiers comme des jeunes. Avec ce deuxième niveau d'explication, on garde à l'esprit que les interactions locales ne se déroulent pas dans le vide social : la prise en compte du contexte de la France des années 1980-2000 permet de compléter l'analyse des effets pervers du partage de la sécurité. 


\section{Conclusion : identifier les enjeux des acteurs de la sécurité}

Le développement du secteur marchand de la sécurité privée a beaucoup été interprété en terme de privatisation, c'est-à-dire de remplacement des acteurs publics par des acteurs privés. Sur les deux terrains investigués, la thèse $d u$ remplacement ne tient pas : les acteurs privés assurent des missions que la police a délaissé, par manque de moyens et par culture professionnelle. En étudiant le travail des agents de sécurité, des associations et de la police, on a montré que les missions de chaque acteur étaient structurées par des enjeux différents. L'État a des enjeux d'ordre public et demande à sa police de mener des patrouilles de dissuasion et des contrôles d'identité. La stratégie mise en œuvre par la police correspond à la définition de la sécurité comme ordre public. Les personnes visées sont les contrevenants au droit; les étrangers en situation irrégulière en constituent une bonne part. Les gares et les centres commerciaux définissent la sécurité comme un élément de leur politique commerciale. Ils cherchent moins à faire une démonstration de force qu'à assurer une forme de tranquillité propice à la consommation et à la fluidité du lieu. En conséquence, les moyens engagés le sont au profit de politiques de médiation et de traitement social des problèmes épineux.

Ces conceptions différentes de la sécurité ne sont pas forcément contradictoires. Il est évident que la SNCF et la direction du centre commercial profitent de la présence des policiers. Mais la façon spécifique qu'ont les policiers de travailler - l'accent mis sur l'ordre public au détriment des relations avec le public - et leur incapacité à faire évoluer leurs missions face aux évolutions de l'environnement contraignent les entreprises commerciales à mettre en œuvre leur propre définition de la sécurité. La prise en compte des enjeux spécifiques des acteurs impliqués permet de montrer que le problème de la «privatisation » de la sécurité est bien mal posé. Les acteurs non-étatiques ne remettent pas en cause le monopole de la police moderne, puisqu'ils ne produisent pas la même sécurité, et pas pour les mêmes fins : les policiers se spécialisent dans les infractions à la loi (law enforcement), tandis que les agents de sécurité sont préoccupés par la qualité de vie (order maintenance).

La tendance naturelle de la police française est la spécialisation du travail policier autour du maintien de l'ordre et des aspects strictement juridiques de la gestion de l'ordre local. La logique de ce système, dont le fondement est le système nationalisé et centralisé de recrutement et de gestion des carrières, est d'éloigner la police de la population, afin de se prémunir des dérives clientélistes et de la corruption. Cet article suggère que l'arrivée de nouveaux acteurs (notamment privés) dans le champ de la sécurité accompagne et favorise cette pente. Le renforcement de cette tendance semble particulièrement nuisible pour la légitimité de l'institution policière auprès des populations qui sont le plus concernées par un rapport fréquent avec les policiers.

\section{Bibliographie}

Bachmann C. et N. Le Guennec 1997, Autopsie d'une émeute. Histoire exemplaire du soulèvement d'un quartier, Paris : Albin Michel

Bayley D. H. et C. D. Shearing, 1996, «The Future of Policing », Law and Society Review, 30 (3) 
Bayley D. H. et C. D. Shearing, 2001, The New Structure of Policing. Description, Conceptualization, and Research Agenda, Washington : U.S. Department of Justice, National Institute of Justice

Bézès P. 2005, «Le modèle de "l'Etat-Stratège «: genèse d'une forme organisationnelle dans l'administration française », Sociologie du travail, 4, 2005, pp. 431-450

Bézès P. 2006, «Publiciser et politiser la question administrative : généalogie de la réforme néo-libérale de l'Etat dans les années $1970 »$, Revue française d'administration publique, $\mathrm{n}^{\circ} 120,2006$, pp. 721-742

Bickford S., 2000, « Constructing Inequality : City Spaces and the Architecture of Citizenship », Political Theory, 28 (3), June

Bowie K., 1996, « De la gare du XIXe siècle au lieu-mouvement. Évolution ou rupture ? », Annales de la recherche urbaine, $\mathrm{n}^{\circ} 71$, juin, pp. 15-23

Bowling B. et J. Foster, 2002, «Policing and the Police », in Mike Maguire, Rod Morgan et Robert Reiner (dir.), The Oxford Handbook of Criminology, Oxford : Oxford University Press, $3^{\mathrm{e}}$ edition

Chardeau M.C., non daté, "Società delle ferrovie francesi (SNCF) e azioni di solidarietà », in Le persone senza dimora e i luoghi dell'esclusione sociale : le stazioni ferroviarie. Un progetto transnazionale per conoscere e definire azione volte a combattere l'esclusione sociale, Rapport de recherche pour la CARITAS et l'Union européenne

Cohen L., 1996, «From Town Center to Shopping Center: The Reconfiguration of Community Marketplaces in Postwar America », American Historical Review, 101 (4), October

Damon J., 2003, «Cinq variables historiques caractéristiques de la prise en charge des SDF », Déviance et société, 27 (1), pp. 29-31

Dubet F. 1987, La galère. Jeunes en survie, Paris : Fayard, «Points »

Flusty S., 2001, "The Banality of Interdiction: Surveillance, Control and the Displacement of Diversity », International Journal of Urban and Regional Research, 25 (3)

Friedberg E., 1993, Le pouvoir et la règle. Dynamiques de l'action organisée, Paris : Le Seuil, « Points Essais»

Geremek B., 1987, La potence ou la pitié. L'Europe et les pauvres du Moyen Age à nos jours (1978), Paris : Gallimard, « Bibliothèque des histoires », 1987

Goss J., 1993, «The « Magic of the Mall»: An Analysis of Form, Function, and Meaning in the Contemporary Retail Built Environment », Annals of the Association of American Geographers, 83 (1), March

Guélaud C., 2002, «La SNCF : une stratégie de croissance, un imaginaire du déclin », in Pierre-Éric Tixier, Du monopole au marché. Les stratégies de modernisation des entreprises publiques, Paris : La découverte, «Textes à l'appui »

Hassenteufel P. et B. Palier 2005, «Les trompe-l'œil de la gouvernance de l'assurance maladie. Contrastes franco-allemands», Revue Française d'Administration Publique, $\mathrm{n}^{\circ} 113$, p.13-28

Hug P., 2000, «Les agents de sécurité noirs. Un exemple de discrimination dans le monde de la sécurité $»$, Les cahiers de la sécurité intérieure, ${ }^{\circ} 40,2^{\text {ème }}$ trimestre

Jackson K.T., 1996, «All the World's a Mall : Reflections on the Social and Economic Consequences of the American Shopping Center», American Historical Review, 101 (4), October 
Jobert B. (dir.) 1994, Le tournant néo-libéral en Europe, Paris : L'Harmattan

Jobard F. 2002, Bavures policières ? La force publique et ses usages, Paris : La Découverte, «Politique et sociétés »

Le Galès P. 2003, Le retour des villes européennes. Sociétés urbaines, mondialisation, gouvernement et gouvernance, Paris : Presses de Sciences Po

Lepoutre D. 1997, Cours de banlieue. Codes, rites, langages, Paris : Odile Jacob

Lindblom C.E. 1959, «The Science of Muddling Through», Public Administration Review, 19 (2), pp. 79-88

Loader I. et N. Walker, 2001, "Policing as a public good: Reconstituting the connections between policing and the state », Theoretical Criminology, 5 (1), pp. 9-35

Macé É. et A. Peralva 2002, Médias et violences urbaines. Débats politiques et construction journalistique, Paris : La documentation française / IHESI

Monjardet D., 1996, Ce que fait la police. Sociologie de la force publique, Paris : La Découverte, «Textes à l'appui »

Moore M.H., 1992, «Problem-Solving and Community Policing», Crime and Justice, vol. 15, 1992

Ocqueteau F., 2004, Polices entre État et marché, Paris : Presses de la FNSP, «Gouvernances »

Palier B. 2005, Gouverner la sécurité sociale. Les réformes du système français de protection sociale depuis 1945, Paris : PUF, Quadrige Essais-débats

Paolucci G., 2003, "Il mercato della paura in Italia », in Giandomenico Amendola (dir.), Il governo della città sicura. Politiche, esperienze e luoghi communi, Napoli : Liguori

Ribeill G., 1996, «Les métamorphoses de la grande gare française », Annales de la recherche urbaine, $\mathrm{n}^{\circ} 71$, juin

Roché S., 2004, «Vers la démonopolisation des fonctions régaliennes: contractualisation, territorialisation et européanisation de la sécurité intérieure », Revue Française de Science Politique, 54 (1), 2004, pp. 43-70

Roché S., 2005, Police de proximité : nos politiques de sécurité, Paris : Seuil

Rybczynski W., 1993, « The New Downtown », Atlantic Monthly, 271 (5), May

Scheerer S. et H. Hess, 1997, «Social Control : a Defence and Reformulation », in Roberto Bergalli et Colin Sumner (dir.), Social Control and Political Order. European Perspectives at the End of the Century, London : Sage

Sherman L.W., 1995, «Public Regulation of Private Crime Prevention », Annals of the American Academy of Political and Social Sciences, Vol. 539, May

Simon H. 1982, Models of Bounded Rationality. Volume 1 and 2, Boston, MA: MIT Press

South N., 1994, «Privatizing Policing in the European Market : Some Issues for Theory, Policy, and Research», European Sociological Review, 10 (3), December

Soutrenon E., 2001, «Faites qu'ils (s'en) sortent. A propos du traitement réservé aux sans-abri dans le métro parisien », Actes de la recherche en sciences sociales, $\mathrm{n}^{\circ} 136-137$, pp. $38-48$

Weber M., 1963, «Le métier et la vocation d'homme politique », in Le savant et le politique, Paris : UGE, «Bibliothèques 10/18 »

Wilson J.Q. et Kelling G., 1982, «Broken Windows: The Police and Neighborhood Safety », Atlantic Monthly, 249 (3), March, pp. 29-38 